\title{
Temperature Aware Online Algorithms for Minimizing Flow Time ${ }^{\hbar}$
}

\author{
Martin Birks ${ }^{\mathrm{a}}$, Stanley P. Y. Fung, ${ }^{\mathrm{a}, *}$ \\ ${ }^{a}$ Department of Informatics, University of Leicester, Leicester LE1 7RH, United Kingdom
}

\begin{abstract}
We consider the problem of minimizing the total flow time of a set of unit sized jobs in a discrete time model, subject to a temperature threshold. Each job has its release time and its heat contribution. At each time step the temperature of the processor is determined by its temperature at the previous time step, the job scheduled at this time step and a cooling factor. We show a number of lower bound results, including the case when the heat contributions of jobs are only marginally larger than a trivial threshold. Then we consider a form of resource augmentation by giving the online algorithm a higher temperature threshold, and show that the Hottest First algorithm can be made 1-competitive, while other common algorithms like Coolest First cannot. Finally we give some results in the offline case.
\end{abstract}

Keywords: Online algorithms, scheduling, temperature, flow time

\section{Introduction}

Motivation. Green computing is not just trendy, but is a necessity. For example, data centers around the world consume an enormous amount of energy. Very often, this energy consumption and the associated issue of heat dissipation is the biggest factor affecting system design from data centers to handheld devices. Many ways to tackle the issue have been explored. Among them, the design of energy-efficient algorithms is an active area of research; we refer to [1] for an introduction.

In this paper we are interested in controlling the temperature of a microprocessor. Temperature is an important issue in processor architecture design: high temperature affects system reliability and lifespan, but a powerful processor inevitably comes with a high energy consumption and hence high temperature. It was proposed in [7] that, instead of slowing down the processor to control the temperature, one can use proper scheduling algorithms to help as well. Since

\footnotetext{
A preliminary version of this paper appeared in the 10th Annual Conference on the Theory and Applications of Models of Computation (TAMC), 2013.

* Corresponding author.
} 
then a number of papers $[2,4,7]$ have worked on this model. We explain the model below.

The Model. Time is split into discrete time steps. For an integer $t$, we refer to the time interval between the time instants $t$ and $t+1$ as the time step $t$. A total of $n$ jobs arrive. Each job $J$ has a release time $r_{J}$, a heat contribution $h_{J}$, and a unit length processing time. All release times are integers. Thus each job fits into one time step. The temperature of a processor changes depending on the heat contribution of the jobs it executes, and a cooling factor $R>1$ : specifically, if at time $t$ the temperature is $\tau_{t}$ and a job $J$ is executed at this time step then the temperature at the next time step is given by $\tau_{t+1}=\frac{\tau_{t}+h_{J}}{R}$. This is a discrete approximation of the actual cooling which is a continuous time process governed by Faraday's Law, and the unit length jobs represent slices of processes given to a processor. The initial temperature can be set at 0 without loss of generality. The temperature can never exceed the temperature threshold $T$. This can be set to 1 without loss of generality. A job $J$ is therefore admissible at time $t$ if $\tau_{t}+h_{J} \leq R$. This means that any job with $h_{J}>R$ can never be admissible; without loss of generality we thus assume all jobs have $h_{J} \leq R$.

One way of quantifying the performance of temperature-aware scheduling algorithms is to optimize some Quality of Service (QoS) measure subject to the temperature threshold. Arguably the most widely used QoS measure for processor scheduling is the flow time (or response time). The flow time of a job $J$ is defined as the difference between its release time and its completion time. We can consider the total (or average) flow time of all jobs, or the maximum flow time. In this paper we focus on the total flow time.

The scheduling algorithm is online, i.e. it is not aware of jobs not yet released. This is of course a natural way to model jobs arriving at a microprocessor. We use the standard competitive analysis to analyze the effectiveness of online algorithms: an online algorithm $\mathcal{A}$ is $c$-competitive if the objective value returned by $\mathcal{A}$ (for a minimization problem) is at most $c$ times that of an offline optimal algorithm $\mathcal{O}$, on all input instances.

There are several common and simple algorithms that can be used in this temperature model:

- Coolest First (CF): at every time step, schedule the coolest job among all admissible jobs, breaking ties arbitrarily.

- Hottest First (HF): at every time step, schedule the hottest job among all admissible jobs, breaking ties arbitrarily.

- FIFO: at every time step, schedule the job released earliest among all admissible jobs, breaking ties arbitrarily.

They all belong to a natural group of algorithms called non-idling algorithms, i.e., they do not idle when they have an admissible job pending. This is a weaker notion than that of reasonable algorithms as defined in [7], as reasonable algorithms are non-idling algorithms, but with stricter restrictions on which job must be scheduled. 
Related Work. Without temperature constraints, the flow time problem is wellstudied. It is well known that the SRPT (shortest remaining processing time first) algorithm is 1-competitive with preemption. Since in our case all jobs are of unit length, there is no issue of preemption and all pending jobs have the same 'remaining' processing time; thus in the case without temperature constraints any non-idling algorithm is 1-competitive.

With temperatures, we are not aware of any prior work on flow time, although there were research on other objective functions. One of them is to maximize throughput when each job has a deadline. This was shown to be strongly NP-hard in the offline case [7], even if all jobs have identical release times and identical deadlines. They further showed that in the online case, all 'reasonable' algorithms are 2-competitive for $R=2$ and this is optimal. When all jobs have identical release times and identical deadlines, CF was shown to have an approximation ratio between 1.3333 and 1.3889 [8]. The model was generalised to all range of $R$ [4], the weighted jobs case [5] and the longer jobs case [6]. It was observed in [7] that their proof also showed strong NP-hardness for minimizing the maximum or total flow time.

Bampis et al. [2] considered the objective of minimizing the makespan on $m>1$ processors when all jobs are released at time 0 . They presented a generic $2 \rho$-approximate algorithm using a $\rho$-approximate algorithm for classical makespan scheduling as a subroutine, and a lower bound of $\frac{4}{3}$ on the approximability. For a single processor the algorithm gives an approximation ratio of 2. They also considered other objectives when there is no temperature threshold: they minimize the maximum temperature or the average temperature of the schedule instead (subject to a bound on the finishing time of jobs).

Our Contributions. We consider three different cases in this paper:

(1) Bounded job heat: Since the problem is trivial without temperature constraints, it is tempting to believe that the problem is still tractable when the jobs are not very hot; we therefore consider limiting the maximum permissible heat of a job, $h_{\max }$. When $h_{\max }$ is allowed to be exactly $R$ then it can be trivially shown that no algorithm can give a bounded competitive ratio. On the other hand, if $h_{\max } \leq R-1$ then it can be easily shown that any algorithm is 1-competitive (details are in Section 3 ). Therefore we consider the case where $h_{\max }=R-\epsilon$ for some $0<\epsilon<1$. Unfortunately it turns out that positive results remain rather unlikely. The problem remains NP-hard, and we show that the competitive ratio approaches infinity as $\epsilon$ approaches 0 . We show even worse results for non-idling algorithms, that they have an unbounded competitive ratio for any $\epsilon<1$.

(2) Increased temperature threshold: In view of the above, we instead give online algorithms a bit more power by allowing them to have a higher temperature threshold of $1+\epsilon$ while the offline algorithm still has a threshold of 1 . This can correspond to the case where, for example, new tech- 
nologies make the system more resistant to higher temperatures. This kind of 'resource augmentation' concept was first introduced in $[9,11]$.

Note that when $\epsilon \geq \frac{1}{R-1}$ a 1-competitive upper bound is trivial (see Section 4). In this model we can give a positive result: HF is 1-competitive if $\epsilon \geq \frac{R^{2}+R+1}{(R-1)(R+1)^{2}}$. We also show a number of lower bounds as in the bounded job heat model; in particular we show that CF cannot be even constant competitive given any non-trivial higher threshold. This is in stark contrast with the throughput case [7] where CF is optimal but HF can be shown to be not.

For easier illustration, consider the case $R=2$ : our results show that HF is 1-competitive whenever $\epsilon \geq \frac{7}{9}$ (any non-idling algorithm is trivially 1-competitive if $\epsilon \geq 1$ ), while there are no 1-competitive algorithms whenever $\epsilon<\frac{1}{8}$.

(3) The offline complexity: We show that no polynomial-time algorithms can have an approximation ratio better than $O(\sqrt{n})$. We also give an approximation algorithm with approximation ratio 2.618 for the case where all jobs have release time 0 . (Recall that the problem remains strongly NP-hard in this case [7].)

Throughput the paper, $R$ is assumed to be a constant whenever asymptotic notation is used.

\section{Preliminaries}

Notations. We denote the offline optimal algorithm by $\mathcal{O}$ and the online algorithm being considered by $\mathcal{A}$. They will also denote the schedules of the algorithms whenever this is possible without confusion. $f(J)$ denotes the flow time of a job $J$; similarly $f(A)$ and $f(\mathcal{A})$ denote the total flow time of the jobs in a set of jobs $A$ or a schedule $\mathcal{A}$. We use $\tau_{t}$ and $\tau_{t}^{\prime}$ to denote the temperatures of $\mathcal{A}$ and $\mathcal{O}$, respectively, at time $t$. We describe jobs that are pending for an algorithm as being stored in a queue. We denote the queues of $\mathcal{A}$ and $\mathcal{O}$ at time $t$ as $Q_{t}$ and $Q_{t}^{\prime}$ respectively. This refers to the time instant when all jobs that are released at $t$ have arrived, but before any jobs have been scheduled for that time step. We occasionally use $t^{-}$to denote the time instant $t$ just before the jobs with release time $t$ arrives. The number of jobs in $Q_{t}$ is denoted as $\left|Q_{t}\right|$. We drop the subscript $t$ if we are referring to the queue in general and not a particular time step.

Job naming convention. We now describe a job naming convention that we use. For brevity we sometimes refer to a job with heat contribution $h$ as an $h$-job, e.g. a 1-job is a job with heat contribution 1. In particular we will most often use 0 -jobs and $(R-1)$-jobs; for convenience we often simply refer to $(R-1)$-jobs as $B$-jobs (for Bounded heat, as they are the hottest jobs that are still always admissible and always heat up the processor). We observe that 
Observation 1. $A B$-job is always admissible, and the temperature does not decrease after scheduling a $B$-job.

Proof. If a $B$-job is scheduled at $t$, then as $\tau_{t} \leq 1$ we have $\tau_{t+1}=\left(\tau_{t}+R-\right.$ $1) / R \leq 1$, so the temperature threshold is not violated. Also $\left(\tau_{t}+R-1\right) / R \geq \tau_{t}$ if and only if $\tau_{t} \leq 1$.

Job blocks. Very often we use many jobs of the same heat contribution released successively. An $h$-block of size $x$ released at time $t$ is a set of $h$-jobs, released one per time step, for $x$ consecutive time steps starting at $t$. In the proofs we often use them to raise or lower the temperatures of the algorithms. For example, if all jobs of a $B$-block of size $x$ are scheduled consecutively then the temperature immediately afterwards is at least $1-\frac{1}{R^{x}}$, which can be made arbitrarily close to 1 with a large enough $x$. Similarly a 0-block of size $x$ reduces the temperature of the algorithm to at most $\frac{1}{R^{x}}$.

Incremental flow times. It is well known that, in a discrete time model, the flow time of a schedule is equal to the sum of the number of pending jobs (i.e. the queue size) at each time step, over all time steps. In many of the proofs we use this approach and bound the number of jobs in the queues in each time step. Sometimes we call this the incremental flow time of this time step. Moreover we can generalize this and refer to the incremental flow time of a time interval $[u, v)$ as the sum of incremental flow times (i.e. queue sizes) of the time steps $u, u+1, \ldots, v-1$.

Repeating phases. In some of the lower bound proofs, the construction involves forcing the $\mathcal{O}$ and $\mathcal{A}$ schedules into a particular structure, called the repeating phase structure. The schedules consist of a (possibly empty) initial stage, a middle stage, and a final stage, with the following properties:

- In the initial stage $\Theta(1)$ jobs are released, and both $\mathcal{O}$ and $\mathcal{A}$ have incremental flow times of $\Theta(1)$ during the stage. At the end of the initial stage, both $\mathcal{O}$ and $\mathcal{A}$ have $\Theta(1)$ jobs in their queues.

- The middle stage consists of $m$ repeating phases, where $m$ can be made arbitrarily large. Each phase is $t=\Theta(1)$ time steps long and with $\Theta(1)$ jobs released. The exact same sequence of jobs are released and scheduled in each phase in both $\mathcal{O}$ and $\mathcal{A}$. Furthermore, at the end of each phase, $|Q|$ is at least one larger than before the phase starts but $\left|Q^{\prime}\right|$ remains the same.

- The final stage has no job release and is just for completion of the pending jobs.

The intuition of introducing such a structure is as follows. We can prove a lower bound if we can force $\mathcal{A}$ to have at least one more job in its queue than that of $\mathcal{O}$ through the release of a sequence of jobs (see, for example, Theorem 5 later). But to prove a good lower bound, we need to make the difference in 
queue lengths as large as possible. As it turns out, often we can 'magnify' this difference as much as we want by repeating the sequence of jobs a sufficient number of times. Since this idea will be used frequently in the rest of this paper, we extracted their common elements into a repeating phase structure and we have the following lemma.

Lemma 2. Suppose the schedules of $\mathcal{O}$ and $\mathcal{A}$ have repeating phase structure. Then the competitive ratio of $\mathcal{A}$ is $\Omega(n)$ where $n$ is the total number of jobs.

Proof. Let $f$ and $f^{\prime}$ be the incremental flow time of $\mathcal{A}$ and $\mathcal{O}$, respectively, of a phase of the middle stage considering only the contribution of the jobs released in that phase. (That is, any jobs from previous phases do not count towards the queue sizes.) Since the schedules are the same for each phase, $f$ and $f^{\prime}$ are the same for each phase. Since there are $\Theta(1)$ time steps and $\Theta(1)$ new jobs released in a phase, both $f$ and $f^{\prime}$ are $\Theta(1)$.

Let $n_{0}$ and $n_{0}^{\prime}$ be the number of jobs in $Q$ and $Q^{\prime}$ respectively right after the initial stage. Again both $n_{0}$ and $n_{0}^{\prime}$ are $\Theta(1)$. Just before the $i$-th phase, $Q$ contains at least $n_{0}+i-1$ jobs, so the incremental flow time for the $i$-th phase for $\mathcal{A}$ is at least $f+\left(n_{0}+i-1\right) t$, as each additional pending job contributes 1 to the incremental flow time in each of the $t$ steps. On the other hand, $Q^{\prime}$ contains $n_{0}^{\prime}$ jobs just before each phase, so its incremental flow time is $f^{\prime}+n_{0}^{\prime} t$. With $m$ repeating phases, the total incremental flow time in the middle stage for $\mathcal{A}$ is therefore at least $\sum_{i=1}^{m}\left(f+\left(n_{0}+i-1\right) t\right)=\Theta\left(f m+n_{0} m t+m^{2} t\right)=\Theta\left(m^{2}\right)$ and for $\mathcal{O}$ it is $\Theta\left(f^{\prime} m+n_{0}^{\prime} m t\right)=\Theta(m)$.

The initial stage contributes $\Theta(1)$ incremental flow time to both $\mathcal{O}$ and $\mathcal{A}$. The final stage also contribute only $\Theta(1)$ incremental flow time for $\mathcal{O}$ since its number of pending jobs remain the same, $\Theta(1)$, after each phase. (For $\mathcal{A}$ the incremental flow time could be larger). The competitive ratio of $\mathcal{A}$ is therefore $\Omega(m)$.

Note that $m=\Theta(n)$ since the initial and final stages have $\Theta(1)$ jobs released, and each of the $m$ phases also has $\Theta(1)$ jobs released. The lower bound therefore follows.

Finally we state an observation.

Observation 3. If the order of jobs in a schedule is fixed (with idle steps being the only possible variation), then it is always preferable to schedule each job as soon as it is admissible.

Proof. It is easy to see that, if a job is preceded by an idle slot, then swapping the job with the idle slot will result in a lower final temperature, assuming the job is admissible at the earlier time. The lower temperature cannot harm the subsequent schedule, and the flow time is improved. Thus, without loss of generality, we can assume each job, following the prescribed order, is scheduled as early as possible. 
In particular, if all current and future jobs have the same heat contribution, then the jobs are effectively identical, so the order does not matter and Observation 3 tells us that it is best not to idle unnecessarily.

Note that this observation does not mean that it is always better to schedule the hottest pending job, nor it is best to never stay idle when there are admissible jobs; that is because there may be jobs arriving later and it may be better to invert the order of the jobs and wait for the later jobs and schedule them first.

\section{Bounded Maximum Job Heat}

First we consider the online case and where the heat contribution of any job is at most $R-\epsilon$ for some $0<\epsilon<1$. This is the only $\epsilon$ range that gives nontrivial results. No algorithm can give a bounded competitive ratio when $h_{\max }$ is allowed to be exactly $R$. This is because after scheduling any job with a nonzero heat contribution, any algorithm will forever have a positive temperature which means that it will never be able to schedule a job with heat $R$, and so such a job will end up with an infinite flow time. If, on the other hand, $h_{\max }$ is restricted to be at most $R-1$ then any job can be scheduled at any time: it follows from Observation 1 that the temperature threshold can never be violated and that all jobs are always admissible. It is therefore equivalent to the case without temperature constraints where any non-idling algorithm is optimal.

Note that the problem remains NP-hard even if $h_{\max }$ is just above $R-1$. See [3] for a proof; we omit it here as it is similar to the original proof in [7].

\subsection{Lower Bounds}

We show that no online algorithm is better than $\Omega(\log (1 / \epsilon))$-competitive.

Theorem 4. For any integer $k \geq 2$, if $h_{\max }=R-\epsilon$ where $\epsilon \leq \frac{R-1}{R^{k}}$ then any deterministic online algorithm is at least $k$-competitive.

Proof. Fix a deterministic online algorithm $\mathcal{A}$. At time 0 release a $B$-job $J_{1}$ and a job $J_{2}$ with $h_{J_{2}}=\frac{R-1}{R^{k-1}}$. $\mathcal{A}$ will eventually start the jobs. Let $t$ be the time the earlier one started. We analyze the two cases.

Case 1: $\mathcal{A}$ starts $J_{1}$ first. Then $\tau_{t+1}=\frac{R-1}{R}$. At time $t+1$ we release another job $J_{3}$ with $h_{J_{3}}=R-\frac{R-1}{R^{k}}$. In order to start $J_{3}$ the temperature of $\mathcal{A}$ must be no higher than $\frac{R-1}{R^{k}}$ and therefore $J_{3}$ only become just admissible at time $t+k$, even if we ignore $J_{2}$. Let $u$ be the time $\mathcal{A}$ starts $J_{3}$.

Case 1a: $u \geq t+k+1$. In this case we gift $\mathcal{A}$ by assuming $J_{2}$ has already been scheduled with no increase to flow time or heat contribution. Starting at time $u+1$, we release a copy of a job $J_{4}$ with $h_{J_{4}}=R-\frac{1}{R^{k-1}}$ every $k$ time steps, for a large enough number of copies. As $\frac{1}{R^{k-1}}>\frac{R-1}{R^{k}}$, this heat contribution is below $h_{\max }$. No other jobs are released. By Observation 3, we can assume each $J_{4}$ job is scheduled as soon as it becomes admissible because they are all identical and there will never be other pending jobs. 
Observe that for a $J_{4}$ job to be admissible the temperature must be no higher than $\frac{1}{R^{k-1}}$, and that they are always admissible after $k-1$ idle steps. We now show that $\mathcal{A}$ indeed needs $k-1$ idle steps before being able to schedule each of these $J_{4}$ jobs. First, $\tau_{u+1}>1-\frac{R-1}{R^{k+1}}$ due to $J_{3}$ (and $\left.J_{1}\right)$. After $k-2$ idle steps, $\tau_{u+k-1}>\left(1-\frac{R-1}{R^{k+1}}\right) / R^{k-2}$ and it can be easily verified that this is greater than $\frac{1}{R^{k-1}}$. Therefore after $k-2$ idle steps $\mathcal{A}$ is still too hot to schedule the first $J_{4}$ job.

Now consider the rest of the $J_{4}$ jobs. The first $J_{4}$ job is scheduled at time $u+k$. If we allow only $k-2$ idle steps, then $\tau_{u+2 k-1}=\left(\tau_{u+k}+h_{J_{4}}\right) / R^{k-1}=$ $\tau_{u+k-1} / R^{k}+\left(R-\frac{1}{R^{k-1}}\right) / R^{k-1}$. This is at least $\tau_{u+k-1}$ if and only if $\tau_{u+k-1} \leq \frac{1}{R^{k-2}}$, which is true noting that $\tau_{u+1} \leq 1$. Since we know $J_{4}$ is not admissible at $u+k-1$, and $\tau_{u+2 k-1} \geq \tau_{u+k-1}, J_{4}$ is also not admissible at $u+2 k-1$. The same argument applies to all subsequent $J_{4}$ jobs and so each $J_{4}$ job requires $k-1$ idle steps before they can be scheduled.

$\mathcal{O}$ schedules jobs $J_{2}, J_{3}$ and $J_{1}$ at $t, t+1$ and $t+2$ respectively. This gives $\tau_{t+3}^{\prime}=1$ and thus $\tau_{u+1}^{\prime} \leq \tau_{t+k+2}^{\prime}=\frac{1}{R^{k-1}}$. $\mathcal{O}$ can therefore schedule each $J_{4}$ job as soon as it is released, reaching a temperature of exactly 1 afterwards, and then repeat the same for the next $J_{4}$ job.

If in total $m$ copies of $J_{4}$ are released then $f(\mathcal{O})=m$ counting only the $J_{4}$ jobs while $f(\mathcal{A}) \geq \mathrm{km}$. As $m$ can be made arbitrarily large, we can ignore the flow time of the first three jobs and get a competitive ratio arbitrarily close to $k$.

Case 1b: $u=t+k$. In this case $J_{2}$ could not have been scheduled before $\overline{J_{3}}$, and $\tau_{u+1}=1$. We release a copy of a job $J_{4}$ with $h_{J_{4}}=R-\frac{1}{R^{k-1}}$ every $k$ steps starting at time $u+2$, for a large enough number of copies. No other jobs are released.

Suppose first that $J_{2}$ is scheduled before any $J_{4}$ job. Then it is best (Observation 3) to schedule $J_{2}$ as early as possible, i.e. at time $u+1$. Then at time $u+k$ the temperature is strictly higher than $\frac{1}{R^{k-1}}$, making $J_{4}$ jobs inadmissible. Therefore the first $J_{4}$ job can be scheduled earliest at $u+k+1$. Similarly to Case 1 a we can argue that all subsequent $J_{4}$ jobs also require $k-1$ idle steps before they can be scheduled.

Now suppose $J_{2}$ is not started before the first $J_{4}$ job, then the first $J_{4}$ job can be (and will be, as it is always better to schedule early) scheduled at $u+k$. Moreover $\tau_{u+k+1}=1$. Therefore all subsequent $J_{4}$ jobs follow the same pattern: if $J_{2}$ remains unscheduled by $\mathcal{A}$ in $[u, u+i k)$, for some $i \geq 1$, then $\tau_{u+i k}=\frac{1}{R^{k-1}}$, and so the next $J_{4}$ job will be scheduled at $u+i k$, giving $\tau_{u+i k+1}=1$. If $J_{2}$ is scheduled in some interval $[u+i k+1, u+(i+1) k+1)$, then the next $J_{4}$ job is not admissible until time $u+(i+1) k+1$. From this point onwards there must again be $k-1$ idle steps between two $J_{4}$ jobs. We will show below that $J_{2}$ cannot be indefinitely delayed.

$\mathcal{O}$ schedules in the same way as in Case 1a. If in total $m$ copies of $J_{4}$ are released after $J_{2}$ is scheduled, then $f(\mathcal{O})=m$ counting only these $J_{4}$ jobs 
while $f(\mathcal{A}) \geq k m$, so again this gives a competitive ratio of $k$. If $J_{2}$ gets postponed after $m J_{4}$ jobs, then the flow time of $J_{2}$ in $\mathcal{A}$ alone is already at least $\mathrm{km}$, so no more $J_{4}$ jobs need to be released and the argument works as well.

Case 2: $\mathcal{A}$ starts $J_{2}$ first at time $t$, then starts $J_{1}$ at time $v \geq t+1$. At time $v+1$ we release a job $J_{3}$ with $h_{J_{3}}=R-\frac{R-1}{R^{k}}$. After $k-1$ idle steps, $\tau_{v+k}>\frac{h_{J_{1}}}{R^{k}}=\frac{R-1}{R^{k}}$. This means that $J_{3}$ cannot be started by $\mathcal{A}$ until $v+k+1$ at the earliest. Let $u$ be the time $\mathcal{A}$ starts $J_{3}, u \geq v+k+1$.

Next we release jobs $J_{4}$ with $h_{J_{4}}=R-\frac{1}{R^{k-1}}$, starting at time $u+1$ and repeating every $k$ time steps for a large number of copies. At $u+1$ the situation of $\mathcal{A}$ is the same as in Case 1a, i.e., due to the heat contribution of $J_{3}$ alone the first $J_{4}$ job requires $k-1$ idle time steps before it can be scheduled. The same argument in Case 1 a for the subsequent $J_{4}$ jobs also applies.

Meanwhile $\mathcal{O}$ starts $J_{2}, J_{3}$ and $J_{1}$ at time $t, t+2$ and $t+3$. As in Case 1 , each $J_{4}$ job will be scheduled immediately by $\mathcal{O}$. Again we can release large enough number of copies to give a competitive ratio of $k$.

The above theorem only gives a non-trivial result when $\epsilon \leq \frac{R-1}{R^{2}}$. The next theorem gives a bound that is not as strong but holds for any $\epsilon<1$.

Theorem 5. Any deterministic algorithm is at least 2 -competitive when $h_{\max }=$ $R-1+\delta$ for any $\delta>0$.

Proof. Fix a deterministic algorithm $\mathcal{A}$. Observe that all we need to do is to force $\mathcal{A}$ into the following situation:

Situation (S): $\left|Q_{t^{-}}\right|=1$ and $\left|Q_{t^{-}}^{\prime}\right|=0$ for some $t$.

Once this happened, a $B$-block of size $b$ is released at time $t$, where $b \gg t$ (an order of magnitude bigger for example). In the next $b$ time steps, $\mathcal{O}$ executes a $B$-job per time step, and its incremental flow time is $b$, while that of $\mathcal{A}$ is at least $2 b$. Thus for large enough $b, f(\mathcal{A})$ is arbitrarily close to $2 f(\mathcal{O})$.

Choose a sufficiently large integer $x$ such that $\alpha=\frac{1}{R^{x}}<R \delta$. At time 0 we release a $B$-block of size $x$. $\mathcal{O}$ schedules these jobs in the next $x$ time steps. $\mathcal{A}$ must do the same: if it stays idle at any point then situation (S) immediately happens. At time $x, \tau_{x}=1-\alpha$, and we then release a $B$-job and another job $J_{1}$ with $h_{J_{1}}=R-1-R \delta+\alpha$. Note that $h_{J_{1}}<R-1$.

If $\mathcal{A}$ idles at time $x$ then $\mathcal{O}$ schedules the two jobs in the next two steps and at time $x+2$ situation (S) happens.

If $\mathcal{A}$ schedules the $B$-job at time $x$ then at time $x+1$ we release a job $J_{2}$ with $h_{J_{2}}=R-1+\delta . \mathcal{O}$ schedules $J_{1}, J_{2}$ and the $B$-job at time $x, x+1$ and $x+2$ respectively. At time $x+1, \mathcal{A}$ is too hot to schedule $J_{2}$ as $\tau_{x+1}=1-\frac{\alpha}{R}>1-\delta$. If $\mathcal{A}$ starts $J_{1}$, then $\tau_{x+2}=1-\delta+\frac{\alpha}{R}-\frac{\alpha}{R^{2}}$ and so $\mathcal{A}$ is still too hot to schedule $J_{2}$ at time $x+2$ and must idle. Alternatively $\mathcal{A}$ could idle at time $x+1$. Either way, we have situation (S) at time $x+3$. 
Finally if $\mathcal{A}$ schedules $J_{1}$ at time $x$, then $\tau_{x+1}=1-\delta$. $\mathcal{A}$ must then schedule the $B$-job at $x+1$ or else $\mathcal{O}$ clears its jobs and situation (S) happens at time $x+2$. This gives $\tau_{x+2}=1-\frac{\delta}{R}$. Meanwhile $\mathcal{O}$ schedules the $B$-job at time $x$ and $J_{1}$ at $x+1$ and end up with a temperature of $\tau_{x+2}^{\prime}=1-\delta+\frac{\alpha}{R}-\frac{\alpha}{R^{2}}$. At time $x+2$ we then release a job $J_{3}$ with $h_{J_{3}}=R-1+\delta-\frac{\alpha}{R}+\frac{\alpha}{R^{2}}$, so that $\mathcal{O}$ can schedule this job while $\mathcal{A}$ cannot (it would lead to $\tau_{x+3}=\left[R+\left(\delta-\frac{\alpha}{R}\right)\left(1-\frac{1}{R}\right)\right] / R>1$ ). Again at time $x+3$ we have situation (S).

\subsection{Non-idling Algorithms}

For the special case of non-idling algorithms we prove a stronger lower bound: it gives an unbounded competitive ratio for all non-trivial values of $h_{\max }$ (recall that a bound of $R-1$ makes every job always admissible.)

Theorem 6. When $h_{\max }=R-1+\delta$ for any constant $\delta>0$, any non-idling algorithm has a competitive ratio of $\Omega(n)$.

Proof. Fix a non-idling algorithm $\mathcal{A}$. Choose a large enough constant integer $b$ so that it is too hot to schedule an $h_{\max }$-job when the temperature is at least $1-\frac{1}{R^{b}}$. It also has to be large enough to satisfy some additional conditions (see Case 1 in sub-phase, and the end of a phase, below.) This value only depends on $R$ and $\delta$. The proof forces the schedules to have repeating phase structure (Section 2), where each repeating phase in turn contains a large number of subphases. Before we describe the overall construction, we begin with a description of sub-phases and phases.

Definition of a sub-phase. Each sub-phase $[u, v)$ contains $b+5$ time steps. We maintain the following properties:

(i) At both $u$ and $v, \mathcal{A}$ 's temperature is at least $1-\frac{1}{R^{b}}$.

(ii) At both $u^{-}$and $v^{-}, Q^{\prime}$ contains only $B$-jobs, $\left|Q_{u^{-}}^{\prime}\right| \geq 1$, and $\left|Q_{v^{-}}^{\prime}\right|=$ $\left|Q_{u^{-}}^{\prime}\right|+1$.

(iii) At both $u^{-}$and $v^{-}, Q$ contains only $h_{\max }$-jobs, and $\left|Q_{v^{-}}\right|=\left|Q_{u^{-}}\right|+1$.

A sub-phase works as follows. At time $u$ we release a job $J_{1}$ with $h_{J_{1}}=$ $R-1-R \delta / 4$ and a job $J_{2}$ with $h_{J_{2}}=R-1-3 R \delta / 4$. Both $J_{1}$ and $J_{2}$ are admissible for $\mathcal{A}$ at $u$ while any $h_{\text {max }}$-jobs are not by definition of $b$, so $\mathcal{A}$ must schedule either $J_{1}$ or $J_{2}$. Consider two cases (see Figure 1):

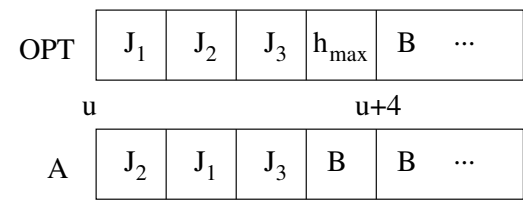

Case 1

OPT \begin{tabular}{|l|l|l|l|l|ll|}
\hline B & $\mathrm{J}_{1}$ & $\mathrm{~J}_{2}$ & $\mathrm{~J}_{3}$ & $\mathrm{~h}_{\max }$ & $\mathrm{B}$ & $\cdots$ \\
\hline
\end{tabular}
\begin{tabular}{|l|l|l|l|l|l|ll|}
$\mathrm{u}$ & $\mathrm{J}_{1}$ & $\mathrm{~J}_{2}$ & B & $\mathrm{J}_{3}$ & B & B & $\cdots$ \\
\hline
\end{tabular}

Case 2

Figure 1: A sub-phase. 
Case 1: $\mathcal{A}$ schedules $J_{2}$ at time $u$. We release the following additional jobs: at $u+2$, a job $J_{3}$ with $h_{J_{3}}=(R-1)\left(1-\delta-\frac{\delta}{4 R}\right)$; at time $u+3$, a $B$-job and an $h_{\text {max }}$-job. $\mathcal{A}$ must schedule $J_{1}, J_{3}$ and the $B$-job at time $u+1, u+2, u+3$ respectively: we show that any $h_{\max }$-jobs are not admissible at any of these time steps. Note that $\tau_{u} \geq 1-\frac{1}{R^{b}}$ can be made arbitrarily close to 1 ; for simplicity of the formulas we first assume $\tau_{u}=1$ here. Then we have:

$$
\tau_{u+1}=1-\frac{3 \delta}{4} ; \quad \tau_{u+2}=1-\frac{\delta}{4}-\frac{3 \delta}{4 R} ; \quad \tau_{u+3}=1-\delta+\frac{\delta}{2 R}-\frac{\delta}{2 R^{2}}
$$

It is easy to check that they are all larger than $1-\delta$. The fact that $\tau_{u}$ is not 1 but $1-\frac{1}{R^{b}}$ means the temperatures are actually slightly lower than those indicated above, but we can choose a large enough $b$ to ensure the difference is small enough so that any $h_{\max }$-job remains not admissible.

Meanwhile $\mathcal{O}$ schedules $J_{1}, J_{2}, J_{3}$ and the $h_{\max }$-job at time $u, u+1, u+2$ and $u+3$. It can be verified that it is indeed feasible.

At $u+4$ we then release a $B$-block of size $b+1$. Both $\mathcal{A}$ and $\mathcal{O}$ schedules these $B$-jobs in the next $b+1$ steps. ( $\mathcal{A}$ is too hot to schedule $h_{\text {max }}$-jobs at any of these steps: it is already the case at $u+3$, and $B$-jobs can only raise the temperature by Observation 1.) At time $v=u+b+5$ the temperature of $\mathcal{A}$ therefore returns to at least $1-\frac{1}{R^{b}}$ (in fact at least $1-\frac{1}{R^{b+1}}$ ). The other properties of the sub-phase are also satisfied at $v$.

Case 2: $\mathcal{A}$ schedules $J_{1}$ at time $u$. At $u+2$ and $u+3$ we release a $B$-job and $J_{3}$ respectively, and at time $u+4$ we release a $B$-job and an $h_{\text {max }}$-job. $\mathcal{A}$ must schedule $J_{2}$, a $B$-job, $J_{3}$ and the other $B$-job at time $u+1$ to $u+4$, in this order: we show that $\mathcal{A}$ is too hot to schedule any $h_{\text {max }}$-job in any of these steps. As before we can assume $\tau_{u}=1$.

$$
\begin{gathered}
\tau_{u+1}=1-\frac{\delta}{4} ; \quad \tau_{u+2}=1-\frac{3 \delta}{4}-\frac{\delta}{4 R} \\
\tau_{u+3}=1-\frac{3 \delta}{4 R}-\frac{\delta}{4 R^{2}} ; \quad \tau_{u+4}=1-\delta+\frac{3 \delta}{4 R}-\frac{\delta}{2 R^{2}}-\frac{\delta}{4 R^{3}}
\end{gathered}
$$

Again it can be verified that $\mathcal{A}$ is hotter than $1-\delta$ at all these times.

Meanwhile $\mathcal{O}$ schedules an existing $B$-job, $J_{1}, J_{2}, J_{3}$ and an $h_{\text {max }}$-job at time $u$ to $u+4$, in this order. It can be verified that this is indeed feasible.

At $u+5$ we then release a $B$-block of size $b$. Again we know that $\tau_{u+5}>$ $\tau_{u+4}>1-\delta$ as $\mathcal{A}$ scheduled a $B$-job at $u+4$, so both $\mathcal{A}$ and $\mathcal{O}$ schedule $B$-jobs in the next $b$ steps. The temperature of $\mathcal{A}$ returns to at least $1-\frac{1}{R^{b}}$ as at time $u$, and that the other properties of a sub-phase are satisfied at time $v$.

Definition of a phase. We maintain the following properties for a phase $[u, v)$ : (i) $\mathcal{A}$ 's temperature is at least $1-\frac{1}{R^{b}}$ at both $u$ and $v$. 
(ii) At both $u^{-}$and $v^{-}, Q^{\prime}$ contains only $B$-jobs, $\left|Q_{u^{-}}^{\prime}\right| \geq 1$, and $\left|Q_{v^{-}}^{\prime}\right|=\left|Q_{u^{-}}^{\prime}\right|$. (iii) At both $u^{-}$and $v^{-}, Q$ contains only $h_{m a x}$-jobs, and $\left|Q_{v^{-}}\right|=\left|Q_{u^{-}}\right|+1$.

Let $y \geq 0$ be the maximum number of $h_{\text {max }}$-jobs that can be scheduled consecutively from a starting temperature between $\left(1-\frac{1}{R^{b}}\right) / R$ and $1 / R$, before it becomes too hot to schedule one more. It is a constant depending on $R$ and $\delta$ only. Note that $y$ may seem ill-defined at first sight as the value may depend on the exact starting temperature. But we can choose a large enough $b$ so that for any starting temperature in that range, the maximum number of consecutive $h_{\max }$-jobs is the same.

A phase $[u, v)$ begins with $y+1$ sub-phases, with the first one starting at time $u$ and each subsequent one immediately following the end of the previous one. Clearly the starting properties of each sub-phase are satisfied because of the starting properties of a phase. Let $t$ be the time when the last sub-phase ends. From the properties of a sub-phase it follows that at $t^{-}, Q^{\prime}$ has gained $y+1$ new $B$-jobs comparing to time $u^{-}$while $Q$ has gained $y+1$ new $h_{\text {max }}$-jobs comparing to time $u^{-}$. No jobs are released for the next $y+1$ time steps. $\mathcal{A}$ is too hot to schedule an $h_{\max }$-job at time $t$ and therefore must idle for one step. At $t+1$ it can then schedule exactly $y h_{\max }$-jobs before it becomes too hot to schedule the next one, by definition of $y$. Therefore $\mathcal{A}$ leaves one new $h_{\max }$-job in $Q$ by time $t+y+1$. Meanwhile $\mathcal{O}$ schedules $y+1 B$-jobs in these time steps, so $Q^{\prime}$ returns to the same number of $B$-jobs as $u^{-}$. Finally at $t+y+1$ we release a $B$-block of size $b$, which both $\mathcal{A}$ and $\mathcal{O}$ schedules, bringing $\mathcal{A}$ 's temperature to at least $1-\frac{1}{R^{b}}$.

The complete construction. The initial stage is as follows. Let $z \geq 1$ be the number of time steps needed to cool down from a temperature of $1-\frac{1}{R^{b}}$ to $1-\delta$ (i.e. it is cool enough to schedule an $h_{m a x}$-job). This is a constant that depends on $R$ and $\delta$ only. At time 0 we release a $B$-block of size $b+z$. $\mathcal{A}$ schedules all these jobs, leaving $\tau_{b+z}=1-\frac{1}{R^{b+z}}$, while $\mathcal{O}$ only schedules the first $b$ of them and idles for the next $z$ steps. At time $b+z$ we release a $B$-job and a $h_{\max }$-job. $\mathcal{A}$ is too hot to schedule the $h_{\max }$-job, so it schedules the $B$-job and so $\tau_{b+z+1}=1-\frac{1}{R^{b+z+1}}$. $\mathcal{O}$ instead schedules the $h_{\max }$-job at time $b+z$, which is admissible by the definition of $z$. At time $(b+z+1)^{-}, Q$ contains only an $h_{\max }$-job while $Q^{\prime}$ have $z+1 B$-jobs. This is the end of the initial stage, and it can be seen that we have established the starting conditions required for a phase.

We then release an arbitrarily large number of phases, each immediately following the preceding one. The number of time steps in a phase is $(b+5)(y+$ $1)+(y+1)+b=\Theta(1)$. By Lemma 2, by releasing $n / \Theta(1)=\Theta(n)$ phases the competitive ratio is at least $\Theta(n)$.

We now show a trivial upper bound. Note that for any constant $h_{\max }$ (i.e. constant $k$ ) this bound is tight (by comparing with Theorem 6).

Theorem 7. For any integer $k \geq 1$, if $h_{\max }=R-\epsilon$ where $\epsilon \geq \frac{1}{R^{k}}$, any non-idling algorithm is $O(k n)$-competitive. 
Proof. Index the jobs $J_{1}, \ldots, J_{n}$ in the order they are completed by $\mathcal{A}$. Note that any job with heat contribution at most $R-\epsilon$ can be scheduled after $k$ idle time steps, and $J_{1}$ must be completed immediately on arrival. Hence $f\left(J_{1}\right)=1$ and $f\left(J_{i}\right) \leq f\left(J_{i-1}\right)+(k+1) \leq(i-1) k+i$ for $i>1$, giving $f(\mathcal{A}) \leq 1+$ $\sum_{i=2}^{n}((k+1) i-k)=O\left(k n^{2}\right)$. On the other hand, $f(\mathcal{O}) \geq n$ as the flow time of each job must be at least 1 . Therefore $f(\mathcal{A}) / f(\mathcal{O})=O(k n)$.

\section{Increased Temperature Threshold}

In this section we consider a form of resource augmentation where the temperature threshold of the online algorithm is increased to $1+\epsilon$ for some $0<\epsilon<\frac{1}{R-1}$ but the temperature threshold of $\mathcal{O}$ remains at 1 . (Note that the maximum heat contribution of a job is not limited any lower than $R$, unlike in Section 3, as this is the hottest that $\mathcal{O}$ can schedule). Similarly to Section 3, a threshold that is too high will be meaningless as it allows any job to be admissible at any time step, meaning that any non-idling algorithm is trivially 1-competitive. A threshold $1+\epsilon$ will allow any job to be admissible if $(1+\epsilon+R) / R \leq 1+\epsilon$, which is equivalent to $\epsilon \geq \frac{1}{R-1}$. We therefore limit $\epsilon$ to $<\frac{1}{R-1}$.

\subsection{Lower Bound}

We can prove the following lower bound on the threshold required to give competitive algorithms, analogous to Theorem 4.

Theorem 8. For any integer $k \geq 1$, if $\epsilon<\frac{R-1}{R^{k+1}}$ then any deterministic online algorithm has competitive ratio at least $k$.

Proof. At time 0 release a $B$-job $J_{1}$, which $\mathcal{A}$ will start at some time $t$. At $t+1$ we release a job $J_{2}$ with $h_{J_{2}}=R$ which will be started by $\mathcal{A}$ at some time $u$. It must be that $u>t+k$ since otherwise the temperature after the execution of $J_{2}$ would be at least $\frac{R-1}{R^{k+1}}+\frac{R}{R}>1+\epsilon$, exceeding the threshold. Meanwhile $\mathcal{O}$ schedules $J_{2}$ and $J_{1}$ at time $t+1$ and $t+2$ respectively.

Starting at time $u+1$, i.e., immediately after $\mathcal{A}$ has completed $J_{2}$, we release a job $J_{3}$ with $h_{J_{3}}=R-\frac{1}{R^{k-1}}$ every $k$ time steps. No other jobs are released. By Observation 3 we can safely assume that each $J_{3}$ job starts as soon as it is admissible in $\mathcal{A}$ because they all have the same heat contribution and they are the only pending jobs.

Consider the first such job at time $u+1$. $\mathcal{A}$ has just completed $J_{2}$ and has a temperature of at least 1 and at most $1+\epsilon$. It can schedule $J_{3}$ at $u+k$ as $\frac{1+\epsilon}{R^{k}}+\left(R-\frac{1}{R^{k-1}}\right) / R \leq 1+\epsilon$. However it would not be able to schedule $J_{3}$ any earlier, i.e., with fewer than $k-1$ idle time steps, if $\frac{1}{R^{k-1}}+\left(R-\frac{1}{R^{k-1}}\right) / R>1+\epsilon$, i.e., $\epsilon<\frac{1}{R^{k-1}}-\frac{1}{R^{k}}$. This latter condition is true because $\frac{R-1}{R^{k+1}}<\frac{1}{R^{k-1}}-\frac{1}{R^{k}}$ for all $R>1$ and the bound the lemma put on $\epsilon$. So $\mathcal{A}$ schedules it at time $u+k$. Meanwhile at $u+1, \mathcal{O}$ has a temperature of at most $\frac{1}{R^{k-1}}$ (with at least $(u+1)-(t+3) \geq k-1$ idle steps after time $t+2)$ and so is able to start $J_{3}$ immediately. 
The same holds for subsequent $J_{3}$ jobs: $\mathcal{O}$ will always have been idle for $k-1$ time steps before each job is released and so will be able to schedule each $J_{3}$ job immediately, while $\mathcal{A}$ will only be able to schedule each job after it has already been released for $k-1$ time steps. If in total $m$ such $J_{3}$ jobs are released then $f(\mathcal{A})=k m+(t+1)+(u-t) \geq k m+t+k+1$, while $f(\mathcal{O})=m+(t+3)+1$. As $m$ can be made arbitrarily large this gives us a competitive ratio arbitrarily close to $k$.

The following is a direct consequence of the above theorem.

Corollary 9. No algorithm can be 1-competitive when $\epsilon<\frac{R-1}{R^{3}}$.

\subsection{Hottest First is 1-competitive}

We now show that HF is 1-competitive when given a sufficiently high threshold, namely $\epsilon \geq \frac{R^{2}+R+1}{(R-1)(R+1)^{2}}$. Note that this threshold is lower than the threshold $\frac{1}{R-1}$ that makes any algorithm 1 -competitive.

We split all jobs into two classes: any job $J$ with $\frac{R^{2}}{R+1}<h_{J}(\leq R)$ is called a hot job, and any other job is called a cool job. We now show three lemmas regarding the properties of hot and cool jobs.

Lemma 10. $\mathcal{O}$ can never schedule two hot jobs consecutively.

Proof. If two hot jobs $J_{1}$ and $J_{2}$ run consecutively, the temperature of $\mathcal{O}$ immediately after running the second job is at least $\frac{h_{J_{1}}}{R^{2}}+\frac{h_{J_{2}}}{R}>\frac{R^{2} /(R+1)}{R^{2}}+$ $\frac{R^{2} /(R+1)}{R}=1$, i.e., it exceeds the temperature threshold.

Lemma 11. If $\epsilon \geq \frac{R^{2}+R+1}{(R-1)(R+1)^{2}}$, then immediately after scheduling a cool job, $\mathcal{A}$ is always cool enough to schedule a hot job.

Proof. A cool job $J_{1}$ and a hot job $J_{2}$ is always able to be scheduled consecutively by $\mathcal{A}$ if $\left(1+\epsilon+h_{J_{1}}\right) / R^{2}+h_{J_{2}} / R \leq 1+\epsilon$, and as $h_{J_{1}} \leq \frac{R^{2}}{R+1}$ and $h_{J_{2}} \leq R$ this is true if $\frac{1+\epsilon+R^{2} /(R+1)}{R^{2}}+\frac{R}{R} \leq 1+\epsilon$, which is equivalent to $\epsilon \geq \frac{R^{2}+R+1}{(R-1)(R+1)^{2}}$.

Lemma 12. If $\epsilon \geq \frac{1}{R^{2}-1}$, a pending cool job is always admissible to $\mathcal{A}$.

Proof. A cool job $J$ is always admissible to $\mathcal{A}$ if $\left(1+\epsilon+h_{J}\right) / R \leq 1+\epsilon$, and as we have that $h_{J} \leq \frac{R^{2}}{R+1}$ this is true if $\left(1+\epsilon+\frac{R^{2}}{R+1}\right) / R \leq 1+\epsilon$, which is equivalent to $\epsilon \geq \frac{1}{R^{2}-1}$.

We refer to the number of cool jobs scheduled by $\mathcal{A}$ and $\mathcal{O}$ in $[0, t)$ (i.e. time steps $0, \ldots, t-1)$ as $c_{t}$ and $c_{t}^{\prime}$ respectively. The number of hot jobs scheduled by $\mathcal{A}$ and $\mathcal{O}$ in $[0, t)$ will similarly be referred to as $h_{t}$ and $h_{t}^{\prime}$.

Lemma 13. If $\epsilon \geq \frac{1}{R^{2}-1}$, and if there exists some time $t$ where $\mathcal{A}$ is idle, it must be that $c_{t}^{\prime} \leq c_{t}$. 
Proof. Consider such a time $t$. $\mathcal{A}$ will not idle if there are pending admissible jobs and by Lemma 12 cool jobs are always admissible. Hence, as $\mathcal{A}$ idles at $t$, it must have scheduled all cool jobs released so far, so $c_{t}^{\prime} \leq c_{t}$.

Lemma 14. If $\epsilon \geq \frac{R^{2}+R+1}{(R-1)(R+1)^{2}}$, then $h_{t}^{\prime} \leq h_{t}$ for all $t$.

Proof. We prove this claim by induction on $t$. First we show two trivial base cases. Before time 0 neither algorithm will have scheduled any job and so $h_{0}^{\prime}=h_{0}=0$. If $\mathcal{O}$ has scheduled a hot job $J$ at time 0 , then this job must also be admissible for $\mathcal{A}$, and as $\mathcal{A}$ always schedules the hottest job possible either $J$ or a hotter job will be scheduled by $\mathcal{A}$ and so $h_{1}^{\prime} \leq h_{1}$.

For a general $t \geq 1$, we use the induction hypotheses $h_{t-1}^{\prime} \leq h_{t-1}$ and $h_{t}^{\prime} \leq h_{t}$ to show that $h_{t+1}^{\prime} \leq h_{t+1}$. Consider the following cases. If $\mathcal{O}$ does not schedule a hot job at $t$ then $h_{t+1}^{\prime}=h_{t}^{\prime} \leq h_{t} \leq h_{t+1}$. If both $\mathcal{O}$ and $\mathcal{A}$ schedule a hot job at $t$ then $h_{t+1}^{\prime}=h_{t}^{\prime}+1 \leq h_{t}+1=h_{t+1}$. The only remaining case to consider is where $\mathcal{O}$ schedules a hot job at $t$ but $\mathcal{A}$ does not. In this case we know by induction that $h_{t-1}^{\prime} \leq h_{t-1}$. By Lemma 10 we know that $\mathcal{O}$ cannot schedule a hot job at $t-1$, so $h_{t+1}^{\prime}=h_{t-1}^{\prime}+1$. Hence, if $h_{t-1}^{\prime} \leq h_{t-1}-1$ then it must be that $h_{t+1}^{\prime}=h_{t-1}^{\prime}+1 \leq h_{t-1} \leq h_{t+1}$.

Otherwise $h_{t-1}^{\prime}=h_{t-1}$. If $\mathcal{A}$ schedules a hot job at $t-1$ then $h_{t+1}=h_{t-1}+1$ and so $h_{t+1}^{\prime}=h_{t-1}^{\prime}+1=h_{t-1}+1=h_{t+1}$. Otherwise $\mathcal{A}$ did not schedule a hot job at $t-1$. However a hot job must be pending at $t$ : as $h_{t-1}^{\prime}=h_{t-1}$ and $\mathcal{O}$ schedules a hot job at $t$, therefore at least $h_{t-1}+1$ hot jobs have been released up to and including time $t$, and as $\mathcal{A}$ does not schedule a hot job at $t-1$, at least one hot job is pending at $t$. $\mathcal{A}$ will always schedule a job when one is admissible though, and we know that $\mathcal{A}$ scheduled either a cool job or no job at $t-1$ so by Lemma 11 this hot job or any hotter admissible job will be scheduled by $\mathcal{A}$ at $t$, contradicting the assumption that it does not.

Theorem 15. If $\epsilon \geq \frac{R^{2}+R+1}{(R-1)(R+1)^{2}}$, HF is 1-competitive.

Proof. Note that $\frac{R^{2}+R+1}{(R-1)(R+1)^{2}}>\frac{1}{R^{2}-1}$ whenever $R>1$. Thus for any $\epsilon \geq$ $\frac{R^{2}+R+1}{(R-1)(R+1)^{2}}$, Lemmas 13 and 14 both hold.

For $f(\mathcal{O})$ to be less than $f(\mathcal{A})$ there must exist some time $s$ such that $\left|Q_{s}\right|>\left|Q_{s}^{\prime}\right|$. Moreover there must also exist some $t<s$ such that $\left|Q_{t}\right|=\left|Q_{t}^{\prime}\right|$, $\mathcal{A}$ idles at $t$ and $\mathcal{O}$ does not idle at $t$. It follows from Lemmas 13 and 14 that if such a time $t$ were to exist, then $c_{t}^{\prime} \leq c_{t}$ and $h_{t}^{\prime} \leq h_{t}$. As $\left|Q_{t}\right|=\left|Q_{t}^{\prime}\right|$, we have that $c_{t}+h_{t}=c_{t}^{\prime}+h_{t}^{\prime}$. Hence the two inequalities must in fact be equalities, i.e. $h_{t}^{\prime}=h_{t}$ and $c_{t}^{\prime}=c_{t}$. We now show that such a $t$ cannot exist, specifically by showing that if $\mathcal{O}$ does not idle at $t$ then $\mathcal{A}$ would not idle either. $\mathcal{O}$ cannot schedule a hot job at $t$, because this means $h_{t+1}^{\prime}=h_{t}^{\prime}+1>h_{t}=h_{t+1}$, contradicting Lemma 14. Suppose $\mathcal{O}$ schedules a cool job at $t$. As $c_{t}^{\prime}=c_{t}$, if a cool job is pending for $\mathcal{O}$ at $t$, then a cool job (not necessarily the same one) must also be pending for $\mathcal{A}$ at $t$. By Lemma 12 we know that this cool job must be admissible for $\mathcal{A}$, contradicting that $\mathcal{A}$ is idle at $t$. 


\subsection{Non-idling Algorithms}

We can show that the more restricted group of non-idling algorithms have unbounded competitive ratio. The general approach of the proof is similar to Theorem 6 .

Theorem 16. For any constant $\epsilon<\frac{1}{R^{2}}$, any non-idling algorithm is $\Omega(n)$ competitive.

Proof. Fix a non-idling algorithm $\mathcal{A}$. We fix some large enough values $b$ and $x$, to be described later. These values depend on $R$ and $\epsilon$ only. Let $X=R-\frac{1}{R^{x}}$. The proof uses a large number of $X$-jobs. Similarly to Theorem 6 , the schedules have repeating phase structure and each phase in turn contains a large number of sub-phases.

Definition of a sub-phase. A sub-phase $[u, v)$ consists of $b+1$ time steps, i.e. $v=u+b+1$, satisfying the following properties:

(i) At both $u$ and $v$ the temperature of $\mathcal{A}$ is at least $1-\frac{1}{R^{b}}$.

(ii) At time $u^{-}, Q^{\prime}$ has one or more $B$-jobs, zero or more 0 -jobs and nothing else. At time $v^{-}, Q^{\prime}$ contains one less $B$-job and one more 0 -job than at time $u^{-}$.

(iii) Both $Q_{u^{-}}$and $Q_{v^{-}}$contain only $X$-jobs, and $\left|Q_{u^{-}}\right|=\left|Q_{v^{-}}\right|$.

The sub-phase works as follows. At $u$ we release a 0 -job. The only other jobs in $Q$ are $X$-jobs, and they are not admissible at $u$ because $\left(1-\frac{1}{R^{b}}\right)+\left(R-\frac{1}{R^{x}}\right)>$ $R(1+\epsilon)$ if $b$ and $x$ are large enough. Hence $\mathcal{A}$ must schedule the 0 -job at this time. This means that $\tau_{u+1} \geq \frac{1}{R}-\frac{1}{R^{b+1}}$. At $u+1$ we release a $B$-block of size $b$. If $\frac{1}{R}-\frac{1}{R^{b+1}}+R-\frac{1}{R^{x}}>R(1+\epsilon)$, then $X$-jobs are not admissible at this time step. This inequality is equivalent to $\epsilon<\frac{1}{R^{2}}-\frac{1}{R^{b+2}}-\frac{1}{R^{x+1}}$. Therefore, as $\epsilon<\frac{1}{R^{2}}$, we can always choose large enough $b$ and $x$ to ensure that $X$-jobs are not admissible. For each time step $u+i$ for $1 \leq i \leq b, \mathcal{A}$ can only (and will) start a $B$-job, as each completed $B$-job will only raise the temperature of $\mathcal{A}$. Meanwhile $\mathcal{O}$ starts one of the $B$-jobs in $Q^{\prime}$ at time $u$, and then run each job from the $B$-block when it is released. It is clear that the sub-phase properties are satisfied at the end of the sub-phase.

Definition of a phase. A phase $[u, v)$ consists of $(b+1) x+x+1+b$ time steps satisfying these properties:

(i) At time $u$ and $v$ the temperature of $\mathcal{A}$ is at least $1-\frac{1}{R^{b}}$.

(ii) At time $u^{-}$and $v^{-}, Q^{\prime}$ contains $x+1 B$-jobs and nothing else.

(iii) Both $Q_{u^{-}}$and $Q_{v^{-}}$contain only $X$-jobs, $\left|Q_{u^{-}}\right| \geq 1$, and $\left|Q_{v^{-}}\right|=\left|Q_{u^{-}}\right|+1$.

A phase $[u, v)$ begins with $x$ consecutive sub-phases, the first starting at time $u$ and then each subsequent sub-phase starting immediately after the end of the previous one. Clearly the properties at the beginning of each sub-phase are satisfied. At $t=u+(b+1) x$ the last sub-phase ends. From the properties of a sub-phase it follows that at time $t^{-}, Q^{\prime}$ contains exactly $x 0$-jobs and one $B$-job, while $Q$ contains the same number of $X$-jobs as at $u^{-}$. 
At $t$ we release a $B$-block of size $x$. Similarly to the situation at a sub-phase, $\mathcal{A}$ must schedule all these $B$-jobs in the next $x$ time steps as the $X$-jobs in $Q$ are still not admissible at any of these time steps, bringing its temperature to at least $1-\frac{1}{R^{x}}$. Meanwhile $\mathcal{O}$ schedules $x 0$-jobs in these time steps bringing its temperature to at most $\frac{1}{R^{x}}$. At time $t+x$, we release an $X$-job. $\mathcal{A}$ is still too hot to schedule $X$-jobs so will idle, while $\mathcal{O}$ schedules the new $X$-job. Finally at $t+x+1$ we release another $B$-block of size $b$ which is scheduled by both $\mathcal{A}$ and $\mathcal{O}$ (again the $X$-jobs are still too hot to be admissible in $\mathcal{A}$ ) bringing the temperature of $\mathcal{A}$ to at least $1-\frac{1}{R^{b}}$. This clearly maintains the phase properties for both $\mathcal{O}$ and $\mathcal{A}$.

The complete construction. At time 0 we start an initial sequence, consisting of a $B$-block of size $b+x+1$ released at time 0 and an $X$-job released at time $b+x$. $\mathcal{A}$ schedules all jobs in the $B$-block, but not the $X$-job because by time $b+x \mathcal{A}$ is already too hot to schedule it. Meanwhile $\mathcal{O}$ schedules the first $b$ $B$-jobs, then idle for $x$ time steps, and schedule the $X$-job at time $b+x$. At time $b+x+1, \mathcal{A}$ is hotter than $1-\frac{1}{R^{b}}$ and $Q$ contains only an $X$-job, while $Q^{\prime}$ contains $x+1 B$-jobs. Thus, we have established the conditions for the start of a phase.

We then release an arbitrarily large number of phases, each immediately following the preceding one. Each phase involves $\Theta(b x)=\Theta(1)$ time steps and jobs. Therefore, by Lemma 2 the competitive ratio of $\mathcal{A}$ is $\Omega(n)$.

Since CF is a non-idling algorithm, Theorem 16 applies, but we can give a stronger bound that shows it has an unbounded competitive ratio for all nontrivial values of $\epsilon$.

Theorem 17. For any constant $\epsilon<\frac{1}{R-1}, C F$ is $\Omega(n)$-competitive.

Proof. Again the proof involves repeating phase structure and sub-phases. Fix some values $b, x$ and $y$ that are all large enough, to be described later. These values depend only on the values of $R$ and $\epsilon$. Let $X=R-\frac{1}{R^{x}}$.

Definition of a sub-phase. Each sub-phase $[u, v)$ lasts $b+x+1$ time steps. We maintain the following properties:

(i) At both $u^{-}$and $v^{-}, Q^{\prime}$ is either empty or contains only 0 -jobs, and $\left|Q_{v^{-}}^{\prime}\right|=$ $\left|Q_{u^{-}}^{\prime}\right|+1$.

(ii) At both $u^{-}$and $v^{-}, Q$ is either empty or contains only $X$-jobs, and $\left|Q_{v^{-}}\right|=$ $\left|Q_{u^{-}}\right|+1$.

A sub-phase contains the release of the following jobs: at time $u$, a $B$-block of size $b$; at time $u+b$, a 0 -block of size $x$; at time $u+b+x$, a 0 -job and an $X$-job.

As $B$ - and 0 -jobs are all cooler than any $X$-jobs that are possibly already in $Q, \mathcal{A}$ schedules the complete $B$-block, followed by the 0 -block and at $u+b+x$ the newly released 0 -job. Meanwhile $\mathcal{O}$ schedules the whole of the $B$ - and 0 -blocks in the same way, but then schedules the $X$-job at $u+b+x$. As $\tau_{u+b+x}^{\prime} \leq \frac{1}{R^{x}}$, the $X$-job is admissible. Clearly, the sub-phase properties are maintained at the end of the sub-phase. 
Definition of a phase. We maintain the properties for a phase $[u, v)$ :

(i) Both $Q_{u^{-}}^{\prime}$ and $Q_{v^{-}}^{\prime}$ are empty,

(ii) Both $Q_{u^{-}}$and $Q_{v^{-}}$contain only $X$-jobs, and $\left|Q_{v^{-}}\right| \geq\left|Q_{u^{-}}\right|+1$.

A phase $[u, v)$ lasts $y(b+x+1)+y$ time steps. It begins with $y$ consecutive sub-phases, the first starting at time $u$, and each subsequent sub-phase starting immediately after the end of the previous one. It is then followed by $y$ time steps with no job release. Clearly the starting condition of a sub-phase is satisfied due to the starting condition of a phase. Let $t=u+y(b+x+1)$ be the time the last sub-phase ends. From the properties of a sub-phase, it follows that at $t^{-} Q^{\prime}$ contains $y 0$-jobs and $Q$ contains at least $y X$-jobs.

Starting at time $t, \mathcal{O}$ schedules the $y 0$-jobs in $Q^{\prime}$ and at time $v^{-}$has an empty queue. We will show that $\mathcal{A}$ however can schedule at most $y-1$ of the $X$ jobs in $Q$ during these $y$ time steps. If this is the case, then the phase properties are satisfied. To show this, note that $\tau_{t}$ must be at least $\left(1-\frac{1}{R^{b}}\right) / R^{x+1}$ because of the structure of a sub-phase. If $\mathcal{A}$ schedules all $y X$-jobs, its temperature at time $v$ is given by

$$
\tau_{v} \geq \frac{1-1 / R^{b}}{R^{x+1+y}}+\sum_{k=1}^{y} \frac{R-1 / R^{x}}{R^{k}}=\frac{1-1 / R^{b}}{R^{x+1+y}}+\left(R-\frac{1}{R^{x}}\right)\left(1-\frac{1}{R^{y}}\right) \frac{1}{R-1}
$$

As all $b, x$ and $y$ can be made arbitrarily large, this can be made arbitrarily close to $\frac{R}{R-1}$. This means if $\epsilon<\frac{1}{R-1}$ the temperature at $v$ will exceed the threshold of $1+\epsilon$, and therefore it is not possible to schedule all $y X$-jobs.

The complete construction. We release an arbitrarily large number of phases, each immediately following the preceding one. Each phase involves $\Theta((b+x) y)=$ $\Theta(1)$ steps and jobs. By Lemma 2, it follows that the competitive ratio of $\mathcal{A}$ is $\Omega(n)$.

Note that Theorems 15 and 17 together imply that HF performs provably better than $\mathrm{CF}$ : $\mathrm{HF}$ is 1-competitive given higher threshold whereas $\mathrm{CF}$ can never be even constant competitive given any non-trivial temperature threshold. This is perhaps somewhat surprising, given that $\mathrm{CF}$ is optimal in maximizing throughput $[4,7]$ but HF can be shown to be not optimal for throughput.

Finally, we consider the algorithm FIFO. FIFO is also a non-idling algorithm, but again we give a stronger bound that shows FIFO has an unbounded competitive ratio for all non-trivial values of $\epsilon$.

Theorem 18. For any constant $\epsilon<\frac{1}{R-1}$, FIFO is $\Omega(\sqrt{n})$-competitive.

Proof. At time 0 we release $x y+10$-jobs for large enough integers $x$ and $y$ to be specified later. Let $X=R-\frac{1}{R^{x}}$. For each $1 \leq i \leq y+1$, at time $i(x+1)-x$ we release an $X$-job. $\mathcal{O}$ schedules all $X$-jobs as soon as they are released, and fills all other slots from time 0 to time $(x+1)(y+1)-x$ with 0 jobs. Meanwhile FIFO schedules all the 0 -jobs first, then schedule the $X$-jobs in $[x y+1, x y+y+2)$ as soon as one is admissible. We are going to show that FIFO must leave a constant fraction of $X$-jobs unscheduled before time $x y+y+2$. 
If FIFO schedules $u X$-jobs consecutively, its temperature immediately afterwards is at least $\left(R-\frac{1}{R^{x}}\right)\left(\frac{1}{R}+\cdots+\frac{1}{R^{u}}\right)=\left(R-\frac{1}{R^{x}}\right)\left(1-\frac{1}{R^{u}}\right) /(R-1)$, which is arbitrarily close to $\frac{R}{R-1}\left(1-\frac{1}{R^{u}}\right)$ if $x$ is large enough. However, the temperature threshold $1+\epsilon$ is strictly less than $\frac{R}{R-1}$. Thus, the maximum number of consecutive $X$-jobs that FIFO can schedule (before it becomes too hot and must idle) is a (large) constant, $u$, independent of $y$.

Therefore, in the time interval $[x y+1, x y+y+2)$ with $y+1$ steps, by choosing a $y \gg u$, at least a constant fraction $(1 /(u+1))$ of these steps are idle. At time $x y+y+2, \mathcal{O}$ has just completed all of the 0 - and $X$-jobs, while FIFO has at least $(y+1) /(u+1) X$-jobs left in its queue. At this point, we release a $B$-block of size $b$, for some arbitrarily large $b$. In each of the next $b$ time steps, $\left|Q^{\prime}\right|=1$ while $|Q|=(y+1) /(u+1)+1$.

We now calculate the flow times of $\mathcal{O}$ and $\mathcal{A}$. For $\mathcal{O}$, all $X$-jobs and $B$ jobs are finished immediately, so have a flow time of $y+1+b$ in total. The total flow time of 0 -jobs is equal to $\sum_{i=1}^{x y+y+2} i-\sum_{i=1}^{y+1}(i(x+1)-x+1)=$ $\Theta\left(x^{2} y^{2}\right)-(2+x y+y+2)(y+1) / 2=\Theta\left(x^{2} y^{2}\right)$. Thus $f(\mathcal{O})=\Theta\left(x^{2} y^{2}+b\right)$.

For $\mathcal{A}$, the total flow time of 0 -jobs is $\sum_{i=1}^{x y+y+2} i=\Theta\left(x^{2} y^{2}\right)$. Since $|Q|=$ $\Theta(y / u)$ for the $b$ steps after time $x y+y+2$, the total flow time of the $B$ jobs is $\Theta(y b / u)$. Thus, even if we ignore the flow time of the $X$-jobs, $f(\mathcal{A}) \geq$ $\Theta\left(x^{2} y^{2}+y b / u\right)$.

Since $n=x y+y+2+b=\Theta(x y+b)$, by setting $x, u$ to be $\Theta(1)$ and $b=y^{2}$, this gives a competitive ratio of $\Theta\left(y^{3} / y^{2}\right)=\Theta(\sqrt{n})$.

\section{The Offline Case}

\subsection{Inapproximability}

In this subsection we prove that the total flow time cannot be approximated with ratio better than $O(\sqrt{n})$. Note that this implies the same lower bound of the competitive ratio of online polynomial-time algorithms, but the proof uses very hot jobs (unlike in Section 3).

The idea of the proof is similar to the $\Omega(\sqrt{n})$ proof in [10]. They considered flow time minimization for jobs of different lengths but without temperature constraints. Essentially, we use very hot jobs to simulate long jobs (since they require a lot of cooling time before they can be scheduled). However, quite intricate technical details are required (for example, we have to ensure that the temperatures are high enough so that the very hot jobs are indeed 'long' jobs).

The proof is a reduction from the NUMERICAL-3D-MATCHING (N3DM) problem. The input to the N3DM problem consists of positive integers $a_{i}, b_{i}$ and $c_{i}, 1 \leq i \leq k$, with $\sum_{i=1}^{k}\left(a_{i}+b_{i}+c_{i}\right)=k D$. The problem is finding if there exists permutations $\pi, \psi$ such that $a_{i}+b_{\pi(i)}+c_{\psi(i)}=D$ for all $i$.

For every N3DM instance and for any given $\epsilon>0$, we construct a corresponding scheduling instance. We first define some numbers: $L=\left\lceil\mu k^{2(1 / \epsilon-1)} D^{1 / \epsilon}\right\rceil$, $M=L k^{2}$, and $N=L M$, where $\mu$ is a large constant. For every number $a_{i}$ in the N3DM instance, we introduce a corresponding job with a heat 
contribution of $R-1 / R^{\left(2 L+a_{i}\right) N-1}$, for every $b_{i}$ a job with a heat contribution of $R-1 / R^{\left(4 L+b_{i}\right) N-1}$, and for every $c_{i}$ a job with a heat contribution of $R-1 / R^{\left(8 L+c_{i}\right) N-1}$. These $3 k$ jobs are called big jobs and they are all released at time $N$.

We will also have a number of jobs that come as $B$-blocks. Each $B$-block, denoted simply by $G(t ; l)$, consists of $l$ jobs, released at times $t+i$ for $i=$ $0, \ldots, l-1$, all with heat contribution $R-1$. Clearly it is always possible to schedule all jobs in $G(t ; l)$ during the time interval $[t, t+l)$ with a total flow time of $l$. We introduce two types of $B$-blocks:

$T_{1}: G(i(14 L+D+1) N ; N)$, for $0 \leq i \leq k$;

$T_{2}: G(k(14 L+D+1) N+N+i L N-2 M ; 2 M)$, for $1 \leq i \leq M$.

This completes the construction of the scheduling instance. See Figure 2.

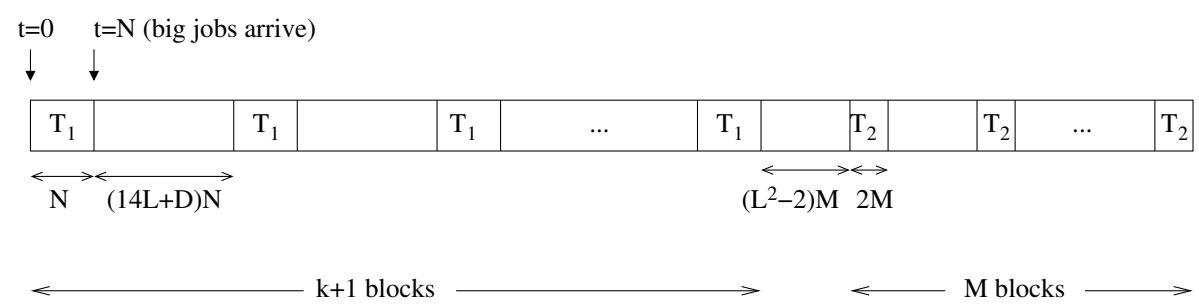

Figure 2: The scheduling instance with $T_{1}$ and $T_{2}$ blocks.

The $k+1 T_{1}$-blocks occur regularly starting at time 0 . Each takes $N$ time steps and leaves $k$ 'holes' each of length $(14 L+D) N$ between them. Similarly, the $T_{2}$-blocks occur regularly, leaving holes of size $\left(L^{2}-2\right) M$ between them (and after the last $T_{1}$-block).

Lemma 19. If the N3DM instance has a solution then for the constructed scheduling instance $f(\mathcal{O}) \leq 52 L M^{2}$ holds.

Proof. Consider the following feasible schedule. All $B$-jobs are scheduled immediately at their release times. All the big jobs are scheduled in the $k$ 'holes' between the $T_{1}$-blocks. For every triple $\left(a_{i}+b_{\pi(i)}+c_{\psi(i)}\right)$ with sum $D$ in the solution, we put the corresponding three jobs together into one of the holes. The job corresponding to $a_{i}$ is scheduled first, followed by the job corresponding to $b_{\pi(i)}$, and finally the job corresponding to $c_{\psi(i)}$.

We now show that such a schedule is feasible. Consider the time right after a $T_{1}$-block. We know that a job of heat contribution $R-\frac{1}{R^{x}}$ is always admissible after $x$ consecutive idle time steps, and so the ' $a$ job' can be completed in $\left(2 L+a_{i}\right) N$ time steps (with $\left(2 L+a_{i}\right) N-1$ idle time steps followed by one time step of actual execution). The same holds for the other two jobs. As there is a matching we know that $a_{i}+b_{\pi(i)}+c_{\psi(i)}=D$. This means that all three jobs can be completed in $(14 L+D) N$ time steps, precisely the size of the hole between two $T_{1}$-blocks. 
All $B$-jobs are scheduled immediately upon release, so their total flow time equals $(k+1) N+2 M^{2}$. The flow time of the three jobs in the $i$-th hole are respectively

$$
\begin{aligned}
& (i-1)(14 L+D+1) N+\left(2 L+a_{i}\right) N \\
& (i-1)(14 L+D+1) N+\left(2 L+a_{i}\right) N+\left(4 L+b_{i}\right) N \\
& (i-1)(14 L+D+1) N+\left(2 L+a_{i}\right) N+\left(4 L+b_{i}\right) N+\left(8 L+c_{i}\right) N
\end{aligned}
$$

So the big jobs yield a total flow time of

$$
\begin{aligned}
& \left(\sum_{i=1}^{k} 3(i-1)(14 L+D+1)+3\left(2 L+a_{i}\right)+2\left(4 L+b_{i}\right)+\left(8 L+c_{i}\right)\right) N \\
= & \left(\frac{3}{2} k(k-1)(14 L+D+1)+\sum_{i=1}^{k}\left(3 a_{i}+2 b_{i}+c_{i}\right)+22 L k\right) N \\
< & (24 L k(k-1)+3 L k+22 L k) N
\end{aligned}
$$

where the last inequality used the facts that $\sum_{i=1}^{k}\left(a_{i}+b_{i}+c_{i}\right)=k D$ and $D \leq L$. Adding in the flow time of the $B$-jobs, this gives an upper bound of

$(k+1) N+2 M^{2}+(25 L k+24 L k(k-1)) N<L M^{2}+2 M^{2}+\left(49 L k^{2}\right) N<52 L M^{2}$.

Next we prove that if the N3DM instance has no solution that the flow time of the corresponding job instance must be large. Before that we need several lemmas on the structure of the optimal schedule regarding how the big jobs and $B$-jobs are scheduled. Define a $T_{1}$-interval to be the time interval during which a $T_{1}$-block is released. $T_{2}$-intervals are defined similarly.

Clearly each block has at least $M$ jobs in it. The number $M$ must be chosen (by choosing a large enough $\mu$ ) such that after scheduling consecutively $M B$ jobs, the temperature of the system is brought 'very close' to 1 . From such a temperature, a big job would require many idle steps before it can be executed, and after it is executed the temperature is again 'very close' to 1 . In fact, when we say 'very close', all we need is that this temperature is strictly greater than $1 / R$; the following lemma makes this precise.

Lemma 20. (i) If at time $t$ the temperature is strictly greater than $1 / R, a$ big job would require at least $2 L N-1$ idle time steps from $t$ before it become admissible.

(ii) There exist a large enough $\mu$ (and hence $L, M$ and $N$ ) so that after executing a big job the temperature is strictly higher than $1 / R$.

(iii) There exists a large enough $\mu$ (and hence $M$ ) so that after a $B$-block of size $M$ is executed the temperature is strictly greater than $1 / R$.

(iv) There cannot be more than one big job scheduled within a $T_{1}-$ or $T_{2}$-interval. 
Proof. (i) If at time $t$ the temperature is strictly greater than $1 / R$, a job with heat contribution $R-\frac{1}{R^{x}}$ would require $x$ idle time steps before it become admissible: trying to schedule the job after $x-1$ idle steps would result in a temperature strictly larger than $\left(\frac{1}{R^{x}}+\left(R-\frac{1}{R^{x}}\right)\right) / R=1$ afterwards. Any big job has heat contribution at least $R-\frac{1}{R^{2 L N-1}}$.

(ii) Clearly, right after scheduling a big job the temperature is at least $1-\frac{1}{R^{2 L N}}$ which is greater than $1 / R$ for large enough $L$ and $N$.

(iii) Since after scheduling $M$ consecutive $B$-jobs the temperature is at least $1-\frac{1}{R^{M}}$, for any constant $R$ there is a corresponding constant $M$ that makes this temperature greater than $1 / R$.

(iv) This follows from (i), (ii) and the fact that a $T_{1}$-interval has length $N$ and a $T_{2}$-interval has length $2 M$.

Lemma 21. In any optimum schedule, and in any subinterval of a $T_{1}$-interval where no big jobs are scheduled, we can without loss of generality assume that the schedule is in the form of a number (possibly zero) of consecutive B-jobs followed by a number (possibly zero) of consecutive idle steps.

Proof. This follows from the same argument as in Observation 3.

Lemma 22. Consider a schedule with flow time less than $N^{2} / 4$ and consider a $T_{1}$-interval $[t, t+N)$. Then

(i) There cannot be more than $N / 2$ idle steps in this $T_{1}$-interval.

(ii) The temperature must be strictly higher than $1 / R$ at some point during $(t, t+N-1]$.

Proof. (i) Suppose there are $y$ idle steps in this $T_{1}$-interval. Then at least $y$ $B$-jobs released here are scheduled after time $t+N-1$. In the best case, the $y$ delayed jobs are the last $y$ released and they are scheduled sequentially in $[t+N, t+N+y)$, so each have a flow time of $y+1$. The $N-y$ non-delayed jobs are scheduled immediately upon arrival and have a flow time of $N-y$. Hence the the total flow time of this block is at least $(N-y)+y(y+1)=N+y^{2}$. Thus, if $y>N / 2$, the flow time of this block alone is already at least $N^{2} / 4$, a contradiction.

(ii) If a big job is scheduled somewhere inside this $T_{1}$-interval then this is true by Lemma 20(ii). Suppose no big job is scheduled in this interval. From (i), at least $N / 2 B$-jobs are scheduled in this interval. By Lemma 21, we can assume they are scheduled consecutively starting at $t$. After $N / 2>M$ such tiny jobs are scheduled consecutively, the temperature is strictly higher than $1 / R$ by Lemma 20(iii).

Lemma 23. Consider a schedule with flow time less than $N^{2} / 4$ and consider a $T_{1}$-interval $[t, t+N)$. Let $t^{*}$ be the last time step within this $T_{1}$-interval with a temperature higher than $1 / R$ (which must exist by Lemma 22(ii)), and let $y_{1}$ be the number of steps after $t^{*}$ until the end of this $T_{1}$-interval, i.e. $t^{*}=t+N-y_{1}$. Then $y_{1}<M$. 
Proof. Clearly no big jobs are scheduled in the last $y_{1}$ time steps. Moreover, by Lemma 21 any $B$-jobs scheduled in these last $y_{1}$ steps, if any, should start from $t^{*}$ consecutively. But any such jobs will raise the temperature, contradicting the definition of $t^{*}$ as the last time step with temperature above $1 / R$. Thus all these last $y_{1}$ time steps must be idle steps. So at least $y_{1} B$-jobs are delayed. Clearly, the only reason to delay scheduling these $y_{1} B$-jobs is to schedule a big job in front of them. As it takes at least $2 L N-1$ idle steps from a temperature of higher than $1 / R$ before a big job is admissible, the big job can finish earliest at time $t+N-y_{1}+2 L N$. Therefore, similarly to the proof of Lemma 22(i), the total flow time of this $T_{1}$-block is at least $\left(N-y_{1}\right)+y_{1}(2 L N)$. If $y_{1} \geq M$ then the flow time of this block is already at least $2 L M N=2 N^{2}$, contradicting the condition of the lemma. Hence $y_{1}<M$.

Lemma 24. Consider a schedule with flow time less than $N^{2} / 4$ and consider a $T_{1}$-interval where a big job is scheduled. Let $y_{2}$ be the number of B-jobs/idle steps in this $T_{1}$-interval before the big job. Then $y_{2}<N / 2+M$.

Proof. By Lemma 21, the $T_{1}$-subinterval before the big job must consist of consecutive $B$-jobs followed by idle steps. There must be less than $M$ consecutive $B$-jobs; any more would result in a temperature of greater than $1 / R$ and the big job cannot be scheduled in this $T_{1}$-interval. By Lemma 22(i), there cannot be more than $N / 2$ idle steps in a $T_{1}$-interval. Hence $y_{2}<N / 2+M$.

Lemma 25. In a schedule with flow time less than $N^{2} / 4$, a big job cannot be scheduled after the end of the last $T_{1}$-interval.

Proof. First we show that none of the big jobs are scheduled between the end of the last $T_{1}$-interval and the end of the last $T_{2}$-interval. By Lemma 23 , the temperature is higher than $1 / R$ at most $M$ steps before the end of the last $T_{1}$-interval. Hence a big job can only be run at least $2 L N-M$ steps after the end of the last $T_{1}$-interval. Since the first $T_{2}$-block arrives after less than $L N$ time steps, and the size of a $T_{2}$-interval is $2 M$, this big job must be executed at least $(2 L N-M)-L N-2 M=L N-3 M$ steps after the end of the first $T_{2}$-interval. Some of the $B$-jobs in this first $T_{2}$-interval may be executed within this $T_{2}$-interval and some may be executed after it. From Lemma 21 we can assume wlog that any $T_{2} B$-jobs not delayed are executed as early as possible in this interval, and those delayed are scheduled after the big job. There are two possibilities: (1) If less than $M B$-jobs are scheduled in the $T_{2}$-interval, i.e. at least $M$ are delayed to after the big job, then the total flow time of these $B$-jobs is at least $(L N-3 M) M=L^{2} M^{2}-3 M^{2} \geq\left(L^{2} / 2\right) M^{2} \geq N^{2} / 4$, contradicting the condition of the lemma. (2) If at least $M B$-jobs are scheduled in the interval, then the temperature becomes higher than $1 / R$, so the big job will need another $2 L N$ idle steps, i.e., it can only be executed after the entire next $T_{2}$-block. We can then apply the same argument to the next block.

Therefore, the only remaining possibility is to execute the big job after the last $T_{2}$-block, which is completed at time $k(14 L+D+1) N+N+M L N \geq$ $N^{2}+N$. Since its release time is $N$, its flow time is therefore at least $N^{2}$, again contradicting the condition of the lemma. 
Lemma 26. If the N3DM instance does not have a solution, then for the constructed scheduling instance $f(\mathcal{O}) \geq N^{2} / 4$ holds.

Proof. Consider an optimum schedule $\mathcal{O}$ and suppose that its total flow time is strictly less than $N^{2} / 4$. From Lemma 25 we know that the big jobs can only be scheduled before the end of the last $T_{1}$-block. First assume all $B$-jobs in $T_{1}$-blocks are scheduled as soon as they are released. Then the big jobs can only be scheduled in the holes between $T_{1}$-intervals. Moreover, the temperature at the beginning of a hole is higher than $1 / R$. Suppose two jobs corresponding to the numbers $c_{i}$ and $c_{j}$ are scheduled into one of these holes. Before each big job can start there must be at least $8 L N-1$ idle time steps (from a point when the temperature is higher than $1 / R$, either because of the $B$-jobs or another big job). Adding the two time steps to actually execute the jobs, there must be at least $16 L N$ time steps in a hole to schedule these two jobs. But each hole is of size $(14 L+D) N$, therefore this is impossible. As there are $k$ holes and $k c$-jobs, having no $c$-job in one hole would imply two or more $c$-jobs in another hole, so it follows that each hole must contain exactly one job corresponding to some $c_{i}$.

However, it could be that some $B$-jobs are not scheduled in their $T_{1}$-intervals and some big jobs are scheduled there instead. From Lemma 23 we know that a temperature higher than $1 / R$ will appear at most $M$ steps before the start of a hole. If a big job is scheduled in a $T_{1}$-interval, we count it towards the hole before it; Lemma 24 tells us that it must be scheduled at most $N / 2+M$ steps into a $T_{1}$-interval. Thus, the 'effective size' of a hole (counting from the point before the hole when the temperature is higher than $1 / R$, to the last point when a big job can be scheduled) is at most $(14 L+D) N+N / 2+2 M \leq 15 L N$, so the argument about the number of $c$-jobs in a hole still works.

Once we have established that each hole has one $c$-job, by similar arguments we can show that every hole must contain exactly one $b$-job, and once that is established we can further show that each hole must contain exactly one $a$-job. Again by relating the heat contribution of the jobs to the number of idle steps required, this implies that for the corresponding three numbers $\left(2 L+a_{i}\right) N+$ $\left(4 L+b_{j}\right) N+\left(8 L+c_{h}\right) N \leq(14 L+D) N+N / 2+2 M$. Moreover, as $a_{i}, b_{j}$ and $c_{h}$ are integers, the quantity on the left is a multiple of $N$ and so the extra $N / 2+2 M$ is not useful and the inequality is equivalent to $\left(2 L+a_{i}\right)+\left(4 L+b_{j}\right)+\left(8 L+c_{h}\right) \leq$ $14 L+D$, i.e. $a_{i}+b_{j}+c_{h} \leq D$. This in turn means that $a_{i}+b_{j}+c_{h}=D$ since the sum of these $3 k$ numbers is $k D$. This gives a solution to the N3DM instance.

We have introduced $3 k$ big jobs, $k+1 B$-blocks of size $N$ and $M B$-blocks of size $2 M$, so the total number of jobs $n$ is equal to $3 k+(k+1) N+2 M^{2}=$ $3 k+(k+1) L^{2} k^{2}+2 L^{2} k^{4}$.

Lemma 27. For any constant $c$ and $\epsilon$, there exists sufficiently large $\mu$ such that $c n^{1 / 2-\epsilon}<L / 208$.

Proof. We have $n=3 k+L^{2} k^{2}(k+1)+2 L^{2} k^{4}<4 L^{2} k^{4}$. Hence

$$
n^{1 / 2-\epsilon}<2 L^{1-2 \epsilon} k^{2-4 \epsilon}<2 L\left(\mu k^{2(1 / \epsilon-1)} D^{1 / \epsilon}\right)^{-2 \epsilon} k^{2-4 \epsilon}=\left(2 \mu^{-2 \epsilon} k^{-2} D^{-2}\right) L .
$$


Hence, no matter how large $c$ is, there is a large enough $\mu$ so that $c n^{1 / 2-\epsilon}$ is smaller than $L / 208$.

Finally we are ready for the proof of the theorem itself.

Theorem 28. For any $\epsilon>0$, there is no polynomial time approximation algorithm for minimizing total flow time with approximation ratio $O\left(n^{1 / 2-\epsilon}\right)$, unless $P=N P$.

Proof. Suppose an approximation algorithm $\mathcal{A}$ with approximation ratio $c n^{1 / 2-\epsilon}$ exists for some $c>0$. The approximation ratio of $\mathcal{A}$ is smaller than $L / 208$ by Lemma 27. Take an instance of N3DM, and perform the above construction. $L$ is polynomial in $k$ and $D$, thus the number of jobs is also polynomial. Moreover, the heat contributions of a big job $R-1 / R^{\left(2 L+a_{i}\right) N-1}$ (more precisely the $-1 / R^{\left(2 L+a_{i}\right) N-1}$ part) can be encoded in $O\left(\left(L+a_{i}\right) N\right)$ bits, again polynomial in $k$ and $D$. Thus, as long as the instance of N3DM is encoded in unary (N3DM remains NP-complete when encoded in unary), the size of the constructed instance is polynomial in the size of the N3DM instance $(\Theta(k D))$ and the construction can be done in polynomial time. Apply algorithm $\mathcal{A}$ to the resulting instance. From Lemma 19, if the N3DM instance has a solution then the flow time of the scheduling instance returned by $\mathcal{A}$ is smaller than $52 L M^{2} \cdot L / 208=N^{2} / 4$. On the other hand, if the N3DM instance has no solution then from Lemma 26 the flow time returned by $\mathcal{A}$ is at least $N^{2} / 4$. Hence with the approximation algorithm $\mathcal{A}$ we can distinguish the two cases and solve the N3DM problem in polynomial time, hence $\mathrm{P}=\mathrm{NP}$.

\subsection{Identical Release Times}

Despite Theorem 28, in the special case of identical release times we can give a 2.618-approximation. Since all release times are 0 , the problem is equivalent to minimizing total completion time, and the algorithm here is similar to the one for minimizing makespan in [2]. As in [2] we assume there is at most one job of heat contribution $R$, otherwise the second job of heat $R$ will have a flow time of infinity for any schedule.

Jobs are indexed in non-decreasing order of heat contribution, i.e., $h_{J_{1}} \leq$ $h_{J_{2}} \leq \ldots \leq h_{J_{n}}$. The jobs are split into two sets depending on their heat contributions: those with heat contributions at most $R-1$ belong to set $\mathcal{C}$, i.e., $\mathcal{C}=\left\{J_{1}, J_{2}, \ldots, J_{c}\right\}$ where $c=|\mathcal{C}|$. All others jobs except the hottest one $\left(J_{n}\right)$ belong to set $\mathcal{H}$, i.e. $\mathcal{H}=\left\{J_{c+1}, J_{c+2}, \ldots, J_{n-1}\right\}$. For simplicity we refer to a job $J_{i}$ in $\mathcal{C}$ (where $i \leq c$ ) as $C_{i}$ and a job $J_{c+i}$ in $\mathcal{H}$ (where $i \leq n-c-1$ ) as $H_{i}$.

The algorithm first assigns the hottest job, $J_{n}$, to the first time step. (If $J_{n} \in \mathcal{C}$, i.e. all jobs are in $\mathcal{C}$, we simply skip this step.) For the next $c$ time steps the algorithm schedules all the jobs in $\mathcal{C}$ in any order. These jobs are always admissible as their heat contributions are at most $R-1$. Finally the jobs in $\mathcal{H}$ are then scheduled in the coolest first order, with each job $H_{i}$ preceded by $k_{i}$ idle time steps, where $k_{i}$ is defined as the largest integer $k$ such that $h_{H_{i}}>\frac{R^{k}-1}{R^{k-1}}$. 
We require two propositions from [2] (generalized to values of $R$ other than 2 ) that are restated here for completeness. The first proposition ensures the schedule described above is feasible.

Proposition 29. Any schedule in which every job $J_{i} \in \mathcal{H}$ is executed after at least $k_{i}$ idle steps is feasible.

Proposition 30. For $R \geq 2$, in an optimal schedule, between the execution of two jobs $H_{j}$ and $H_{i}$ (where $H_{j}$ is before $H_{i}$ ), there are at least $k_{i}-1$ steps which are either idle or execute jobs in $\mathcal{C}$.

Theorem 31. The above algorithm achieves a 2.618-approximation for minimizing total flow time, for $R \geq 2$.

Proof. If $c \geq n-1$ then $\mathcal{A}$ schedules a job every time step without idling and so must be optimal, therefore we only need to consider the case where $c<n-1$. It is clear that $f(\mathcal{A})=f\left(J_{n}\right)+f(\mathcal{C})+f(\mathcal{H})=f(\mathcal{C})+f(\mathcal{H})+1$. For each job $C_{i}, 1 \leq i \leq c$, we have $f\left(C_{i}\right)=i+1$. Therefore $f(\mathcal{C})=\sum_{i=1}^{c} f\left(C_{i}\right)=\frac{c^{2}+3 c}{2}$.

Next we consider each job $H_{i}$ for $1 \leq i<n-c$ : $f\left(H_{1}\right)=1+c+k_{1}+1$ and $f\left(H_{i}\right)=f\left(H_{i-1}\right)+k_{i}+1$. This gives $f\left(H_{i}\right)=c+i+1+\sum_{j=1}^{i} k_{j}$. Thus

$$
f(\mathcal{H})=\sum_{i=1}^{n-c-1} f\left(H_{i}\right)=\frac{n^{2}+n-c^{2}-3 c-2}{2}+\sum_{i=1}^{n-c-1} k_{i}((n-c-1)-(i-1))
$$

The total flow time of $\mathcal{A}$ is therefore

$$
f(\mathcal{A})=1+\frac{c^{2}+3 c}{2}+\frac{n^{2}+n-c^{2}-3 c-2}{2}+\sum_{i=1}^{n-c-1} k_{i}(n-c-i)
$$

We now analyze the flow time of $\mathcal{O}$. Consider an alternative schedule $\mathcal{O}^{*}$ which only schedules jobs in $\mathcal{H} \cup\left\{J_{n}\right\}$ : it puts $J_{n}$ at time 0 , then assigns each job $H_{i}$ in $\mathcal{H}$ a virtual processing time of $k_{i}$, and schedules them according to the order given by the Shortest Processing Time First rule. In each of these $k_{i}$ processing steps, the first $k_{i}-1$ is idle and the last is where $H_{i}$ is executed. By Proposition 30 and the optimality of the Shortest Processing Time First rule for minimizing total flow time when temperature is not considered, $\mathcal{O}^{*}$ must be the optimal way to schedule only jobs in $\mathcal{H} \cup\left\{J_{n}\right\}$.

We denote the flow time in $\mathcal{O}^{*}$ of a job $J$ as $f\left(J^{*}\right)$. Analogous definitions for a set of jobs follow naturally. We have $f\left(H_{1}^{*}\right)=1+k_{1}$ and $f\left(H_{i}^{*}\right)=f\left(H_{i-1}^{*}\right)+k_{i}$ for $1<i<n-c$. This gives $f\left(H_{i}^{*}\right)=1+\sum_{j=1}^{i} k_{j}$. The total flow time for the set $\mathcal{H} \cup\left\{J_{n}\right\}$ is therefore

$f\left(\left(\mathcal{H} \cup\left\{J_{n}\right\}\right)^{*}\right)=1+\sum_{i=1}^{n-c-1} f\left(H_{i}^{*}\right)=1+(n-c-1)+\sum_{i=1}^{n-c-1} k_{i}((n-c-1)-(i-1))$

The total flow time for jobs in $\mathcal{C}$ in the optimal schedule clearly cannot be smaller than $1+2+\ldots+c=c(c+1) / 2$. The flow time of $\mathcal{O}$ cannot be smaller 
than the flow time of optimally scheduling the two sets of jobs $\mathcal{H} \cup\left\{J_{n}\right\}$ and $\mathcal{C}$ separately; hence,

$$
f(\mathcal{O}) \geq \frac{c^{2}+c}{2}+(n-c)+\sum_{i=1}^{n-c-1} k_{i}(n-c-i)
$$

Combining (1) and (2) gives us the approximation ratio:

$$
\frac{f(\mathcal{A})}{f(\mathcal{O})} \leq \frac{n^{2}+n+2 \sum_{i=1}^{n-c-1} k_{i}(n-c-i)}{c^{2}-c+2 n+2 \sum_{i=1}^{n-c-1} k_{i}(n-c-i)}
$$

As $k_{i} \geq 1$ for every $H_{i} \in \mathcal{H}$, it must be that $\sum_{i=1}^{n-c-1} k_{i}(n-c-i) \geq$ $\sum_{i=1}^{n-c-1}(n-c-i)=(n-c-1)(n-c) / 2$. Putting back into the above formula and simplifying, this gives

$$
\frac{f(\mathcal{A})}{f(\mathcal{O})} \leq \frac{c^{2}-2 n c+2 n^{2}+c}{2 c^{2}-2 n c+n^{2}+n}<\frac{c^{2}-2 n c+2 n^{2}}{2 c^{2}-2 n c+n^{2}}
$$

Let $x=c / n$, then this ratio is equal to $\frac{x^{2}-2 x+2}{2 x^{2}-2 x+1}$. For $0 \leq x \leq 1$, the maximum value of this ratio is equal to $(3+\sqrt{5}) / 2$, attained at $x=(3-\sqrt{5}) / 2$.

Note that this algorithm is almost like CF (except the first step), but the change is necessary to obtain a good approximation ratio. Consider the example with two jobs $J_{1}$ and $J_{2}$ with $h_{J_{1}}=R-1$ and $h_{J_{2}}=R-\epsilon$. CF schedules $J_{1}$ first and requires $\log _{R} \frac{R-1}{R \epsilon}$ idle steps before it can schedule $J_{2}$, whereas scheduling $J_{2}$ first followed immediately by $J_{1}$ gives a total flow time of 3 .

\section{Conclusion}

We studied the problem of minimizing total flow time under temperature constraints. Although most of the results are lower bounds, we did show that Hottest First is good in a certain sense (at least for certain ranges of $\epsilon$ ). Some open problems include:

- In the higher threshold model, can we upper-bound the performance of HF in other ranges of $\epsilon$; specifically can it be constant competitive?

- Is there an offline approximation algorithm matching the $\Omega(\sqrt{n})$ lower bound? Without temperatures but with different job lengths, [10] gave an $O(\sqrt{n})$-approximate algorithm, but it does not look like it can be adapted here.

\section{References}

[1] S. Albers. Energy-efficient algorithms. Communications of the ACM 53(5), pages 86-96, 2010. 
[2] E. Bampis, D. Letsios, G. Lucarelli, E. Markakis and I. Milis. On multiprocessor temperature-aware scheduling problems. In Proc. Joint Int. Conf. Frontiers in Algorithmics and Algorithmic Aspects in Information and Management, LNCS 7285, pages 149-160, 2012.

[3] M. Birks, Online algorithms for temperature aware job scheduling problems, PhD Thesis, University of Leicester, 2012.

[4] M. Birks and S. P. Y. Fung, Temperature aware online scheduling with a low cooling factor. In Proc. 7th Annual Conference in Theory and Applications of Models of Computation, LNCS 6108, pages 105-116, 2010.

[5] M. Birks, D. Cole, S. P. Y. Fung and H. Xue. Online algorithms for maximizing weighted throughput of unit jobs with temperature constraints. In Proc. Joint Int. Conf. Frontiers in Algorithmics and Algorithmic Aspects in Information and Management, LNCS 6681, pages 319-329, 2011.

[6] M. Birks and S. P. Y. Fung. Temperature aware online algorithms for scheduling equal length jobs. In Proc. Joint Int. Conf. Frontiers in Algorithmics and Algorithmic Aspects in Information and Management, LNCS 6681, pages 330-342, 2011.

[7] M. Chrobak, C. Dürr, M. Hurand and J. Robert. Algorithms for temperature-aware task scheduling in microprocessor systems. In Sustainable Computing: Informatics and Systems 1(3), pages 241-247, 2011.

[8] C. Dürr, I. Milis, J. Robert and G. Zois. Approximating the throughput by coolest first scheduling. In Proc. 10th Workshop on Approximation and Online Algorithms, LNCS 7846, pages 187-200, 2012.

[9] B. Kalyanasundaram and K. Pruhs. Speed is as powerful as clairvoyance. Journal of the ACM 47(4), pages 617-643, 2000.

[10] H. Kellerer, T. Tautenhahn and G. J. Woeginger. Approximability and nonapproximability results for minimizing total flow time on a single machine. SIAM Journal on Computing 28(4), pages 1155-1166, 1999.

[11] C. A. Phillips, C. Stein, E. Torng and J. Wein. Optimal time-critical scheduling via resource augmentation. In Proc. 29th Annual ACM Symposium on the Theory of Computing, 140-149, 1997.

[12] K. Pruhs, J. Sgall and E. Torng. Online scheduling. In Handbook of Scheduling: Algorithms, Models, and Performance Analysis, chapter 15, pages 15-1 - 15-41. CRC Press, 2004.

[13] J. Yang, X. Zhou, M. Chrobak, Y. Zhang and L. Jin. Dynamic thermal management through task scheduling. In Proc. IEEE Int. Symposium on Performance Analysis of Systems and Software, pages 191-201, 2008. 